\title{
A Rare Case of Broad Ligament Angioleiomyoma
}

\author{
Ujwala Maheshwari $^{1}$, Munot Shweta ${ }^{2}$, Navdeep Kaur ${ }^{2}$, Shilpi Sahu ${ }^{3}$ \\ ${ }^{1}$ Professor, ${ }^{2}$ Post-Graduate Student, ${ }^{3}$ Professor and HOD, \\ Department of Pathology, MGM's Medical College and Hospital, Navi Mumbai.
}

Corresponding Author: Shweta Munot

\begin{abstract}
Angioleiomyoma aka vascular leiomyoma is a benign mesenchymal tumour originating from the smooth muscles and containing thick walled vessels. It is commonly seen in the subcutis of the lower extremities. Although a few cases of uterine angioleiomyoma have been reported, broad ligament angioleiomyoma is extremely rare.

Case History: 45 year old multiparous women presented to the OPD with complains of pain and mass in abdomen since 4 months. No history of menstrual irregularities was noted. Ultrasonography revealed a homogenous solid mass in lower abdomen region. The mass was hypodense. A provisional clinical radiological diagnosis of Broad ligament fibroid was made. The patient underwent a surgery for removal of broad ligament mass with bilateral salpingooophorectomy and specimen was submitted for histopathological examination.
\end{abstract}

Conclusion: Angioleiomyoma of broad ligament is an extremely rare diagnosis and should be diagnosed correctly with the help of IHC markers. It must be kept in mind as a differential diagnosis of abdominal mass associated with pain to be able to arrive at a correct diagnosis.

Keywords: Angioleiomyoma, broad ligament, vascular leiomyoma, mesenchymal tumour, broad ligament fibroid.

\section{INTRODUCTION}

Angioleiomyoma is a benign mesenchymal tumour originating from smooth muscle cells and contains numerous thick-walled blood vessels. It has a very rare occurrence ${ }^{[1]}$. It is commonly found in the skin of lower extremities but is extremely rare in broad ligament. This is one amongst the rarest cases in our tertiary care hospital. Angioleiomyomas are usually seen in the 4 th and 5th decade of life ${ }^{[2]}$. Uterine angioleiomyomas usually present with menorrhagia due changes in uterine vasculature, the presence of venous plexuses and action of growth factors by the tumor cells ${ }^{[2,3]}$. Particular symptoms of the disease point towards hormone-dependent angio leiomyoma formation in women taking hormone replacement therapy (HRT) ${ }^{[3,4]}$. Angioleiomyoma is a unique and a rare lesion as there are no specific clinico radiological findings which help to differentiate it from the more common leiomyoma counterpart; hence, the pathologists have an important role in its diagnosis on basis of histopathological and immunohistochemical examination of the tumour ${ }^{[5]}$.

\section{CASE HISTORY}

45 year old multiparous women resented to the out-patient department with complaints of pain and mass in abdomen since four months. No history of menstrual irregularity was noted.

Ultrasonography revealed a homogenous solid mass in lower abdomen region. The mass was hypodense. A provisional clinical radiological diagnosis of Broad ligament fibroid was made.

The patient underwent a surgery for removal of broad ligament mass with bilateral salpingo-oophorectomy and specimen was submitted for histopathological examination. 


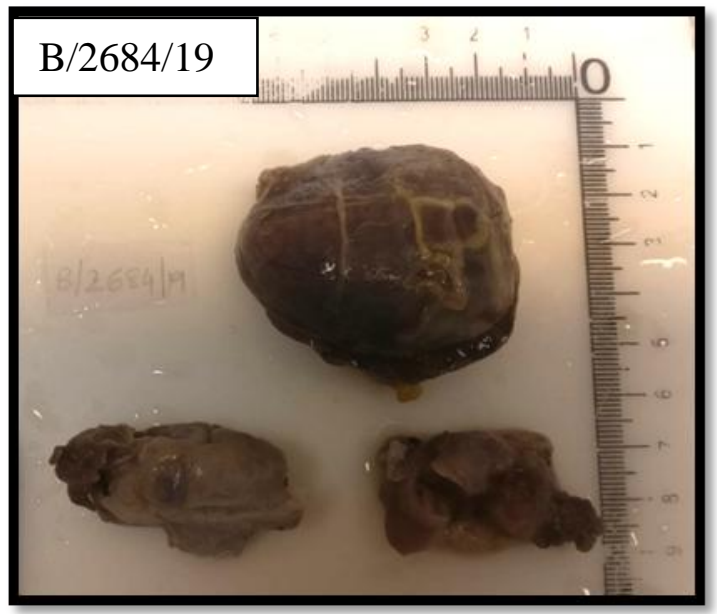

Figure 1: A specimen of a broad ligament mass with bilateral fallopian tubes and bilateral ovaries was received with mass measuring $5.5 \times 4 \times 3.5 \mathrm{~cm}$. External surface was encapsulated and congested.
On gross examination, a specimen of a broad ligament mass with bilateral fallopian tubes and bilateral ovaries was received. Broad ligament mass was measuring $5.5 \times 4 \times 3.5 \mathrm{~cm}$.

External surface was encapsulated and congested.

Cut section was homogenous and grey brown in colour. Separately sent two fallopian tubes measuring $4 \mathrm{~cm}$ and $5 \mathrm{~cm}$ each with attached ovaries measuring $3 \times 2 \times 0.5 \mathrm{~cm}$ and $3.5 \times 2.2 \times 1 \mathrm{~cm}$ respectively.

\section{On microscopic examination,}
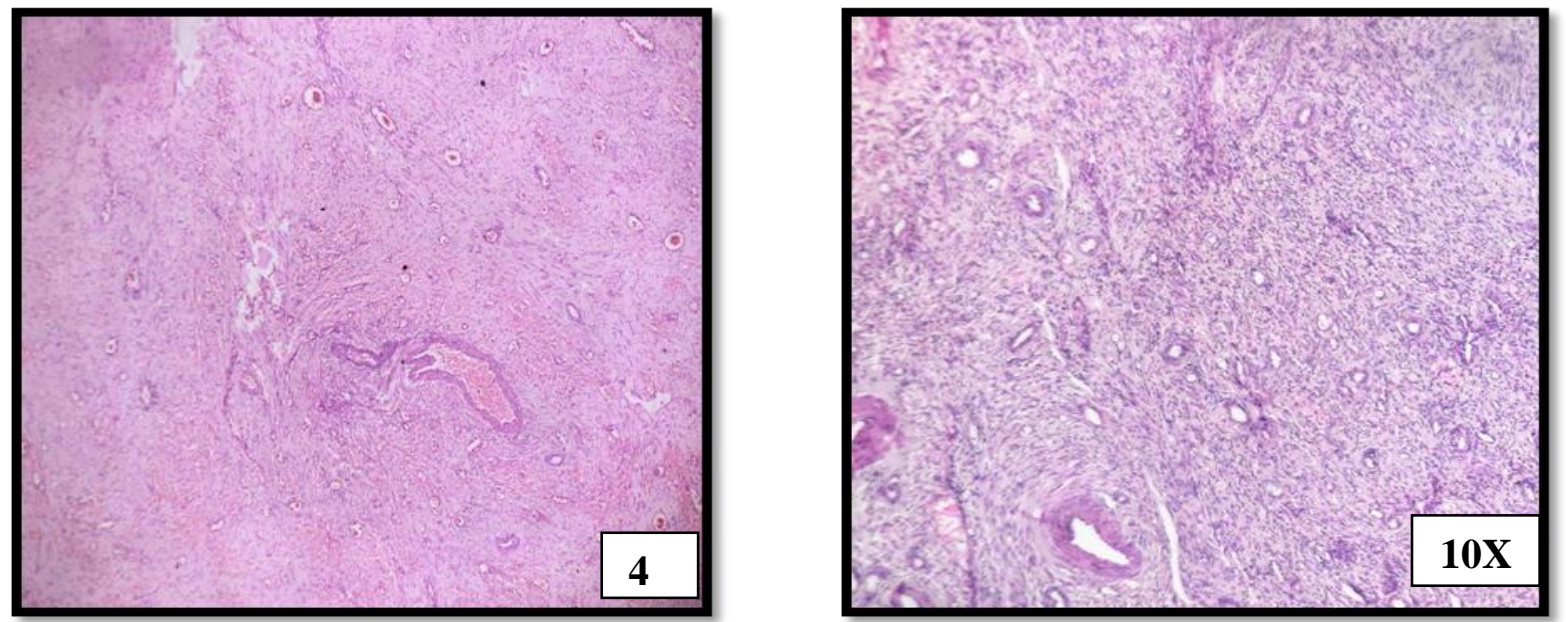

Figure $2 \& 3: \mathrm{H}$ and $\mathrm{E}$ sections reveal well circumscribed lesion comprising fascicles of smooth muscle bundles surrounding vascular lumina which are markedly dialated and congested having thickened walls and are lined by endothelial cells.

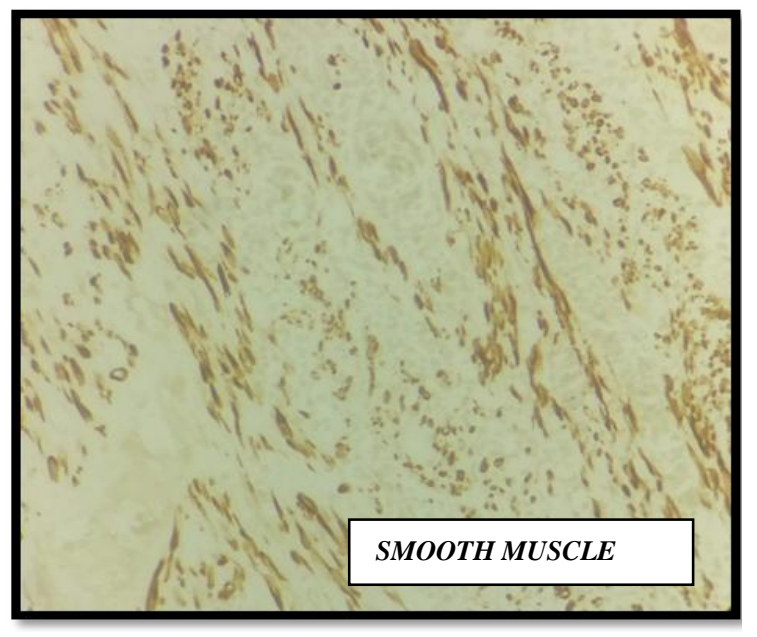

Figure 4: Positive for Smooth Muscle Actin (SMA).

Immunohistochemical markers were done to confirm the diagnosis. The tumour was Positive for Smooth muscle Actin but Negative for Desmin.

\section{DISCUSSION}

Broad ligament angioleiomyoma is rare than its uterine counterpart ${ }^{[1,2]}$. Unlike uterine leiomyomas which arise from parenchymal cells of myometrium, angioleiomyomas originate from the smooth muscle cells present in the wall of blood vessels ${ }^{[2,7]}$. Differential diagnosis of angiofibroma, angiomyolipoma, angiomyofibroblastoma and perivascular epithelioid tumors can be considered. Duhig and Ayer in their study noted that after the surgical excision of the tumour, none of the 
cases showed recurrence ${ }^{[2,8]}$. Repeat computed tomography (CT) scan did not reveal any evidence of recurrence ${ }^{[1]}$. Immunohistochemical markers of smooth muscle cells like as smooth muscle actin (SMA) and blood vessel markers like CD34 and CD31 are quintessential to differentiate angioleiomyoma from its other differential diagnosis like angiofibroma, angiomyolipoma and angiomyofibroblastoma. All these tumors are positive for markers like vimentin and desmin but negative for smooth muscle actin ${ }^{[1,9]}$. The patients with uterine angioleiomyomas commonly present with pain in the abdomen which is due to ischemia because of vascular contraction which can also lead to degenerative changes in the tumour cells ${ }^{[1,}$ 6]. Various complications like spontaneous rupture of the tumor, coagulopathy and pseudo-Meigs syndrome are also noted ${ }^{[2,9}$, 10].

\section{CONCLUSION}

Angioleiomyoma or Vascular leiomyoma is rare variant of uterine fibroid is a benign tumor responsive to surgical excision ${ }^{[2]}$. It needs to be differentiated from the other mesenchymal tumours with predominant vascular structures ${ }^{[2]}$. Uterine angioleiomyomas are associated with a large number complications in its disease course, thus a large number of these requiring blood and blood component transfusion ${ }^{[4]}$. It is a prodigious type of uterine smooth muscle tumour which commonly exhibits particular morphological and immunophenotypical characteristics ${ }^{[5]}$. It is of utmost importance for a pathologist to identify this variant and to distinguish it from its characteristic mimickers, especially a neoplasia where the tumor reveals marked cytologic atypia or increased levels of cancer antigen 125 levels. When needed an immunohistochemical profile should be done to make an accurate diagnosis ${ }^{[1,5,9]}$.

Acknowledgement: None
Conflict of Interest: None

Source of Funding: None

\section{REFERENCES}

1. Angioleiomyoma of Broad Ligament - S Agarwal, S K Gupta, and $\mathrm{N}$ Tejwani. Dept. of Pathology, Lady Hardinge Medical College, NewDelhi, India

2. Hemalatha AL, Nanjundaswamy DN, Sahni S, Kumari A. Angioleiomyoma of Broad Ligament: A Rare Variant of Leiomyoma. Int J Sci Stud 2016;3(11):290-292.

3. Stewart EA, Nowak RA. Leiomyomarelated bleeding: A classic hypothesis updated for the molecular era. Hum Reprod Update 1996;2:295-306.

4. Uterine angioleiomyoma - a rare variant of uterine leiomyoma: review of literature and case reports, Dobrosława L. SikoraSzczęśniak Department of Obstetrics and Gynaecology, Specialist Hospital in Radom, Poland

5. Garima Garg, Sambit K Mohanty. Uterine angioleiomyoma: a rare variant of uterine leiomyoma. Arch Pathol Lab Med. 2014 Aug;138(8):1115-8. doi: 10.5858/arpa.2013-0315-RS.

6. Wang CP, Chang YL, Sheen TS. Vascular leiomyoma of the head and neck. Laryngoscope 2004;114:661-5.

7. Rosai J. Rosai and Ackerman's Surgical Pathology. 10th ed. St. Louis: Mobsy; 2011. p. 1512.

8. Duhig JT, Ayer JP. Vascular leiomyoma. A study of sixty one cases. Arch Pathol 1959;68:424-30.

9. Culhaci N, Ozkara E, Yüksel H, Ozsunar Y, Unal E. Spontaneously ruptured uterine angioleiomyoma. Pathol Oncol Res 2006;12:50-1.

10. Thomas S, Radhakrishnan L, Abraham L, Matthai A. Uterine angioleiomyoma with atypia, raised CA-125 levels, and pseudomeigs syndrome: An alarming presentation. Case Rep Pathol 2012;2012:519473.

How to cite this article: Maheshwari U, Shweta $\mathrm{M}$, Kaur $\mathrm{N}$ et.al. A rare case of broad ligament angioleiomyoma. International Journal of Research and Review. 2021; 8(5): 143-145. DOI: https://doi.org/10.52403/ijrr.20210521 